\title{
Assessment of Health-Related Quality of Life of Tuberculosis Patients in Fako Division, South-West Region of Cameroon
}

\author{
Benjamin D. Thumamo Pokam ${ }^{*}$, Pius Fokam², Theophile Nana Njamen ${ }^{3}$, \\ Prisca Wabo Guemdjom4 ${ }^{4}$ Anne E. Asuquo ${ }^{5}$
}

${ }^{1}$ Department of Medical Laboratory Science, Faculty of Health Sciences, University of Buea, Buea, Cameroon

${ }^{2}$ Department of Surgery, Faculty of Health Sciences, University of Buea, Buea, Cameroon

${ }^{3}$ Department of Obstetrics and Gynecology, Faculty of Health Sciences, University of Buea, Buea, Cameroon

${ }^{4}$ Department of Public Health, Faculty of Health Sciences, University of Buea, Buea, Cameroon

${ }^{5}$ Department of Medical Laboratory Science, Faculty of Allied Medical Sciences, College of Medical Sciences, University of

Calabar, Calabar, Nigeria

Email: *thumamo@yahoo.fr

How to cite this paper: Pokam, B.D.T., Fokam, P., Njamen, T.N., Guemdjom, P.W. and Asuquo, A.E. (2020) Assessment of Health-Related Quality of Life of Tuberculosis Patients in Fako Division, South-West Region of Cameroon. Journal of Tuberculosis Research, 8, 93-110. https://doi.org/10.4236/jtr.2020.83009

Received: May 29, 2020

Accepted: July 11, 2020

Published: July 14, 2020

\section{Copyright $\odot 2020$ by author(s) and} Scientific Research Publishing Inc. This work is licensed under the Creative Commons Attribution International License (CC BY 4.0).

http://creativecommons.org/licenses/by/4.0/

\begin{abstract}
Background: Tuberculosis (TB) caused by Mycobacterium tuberculosis is one of the top 10 causes of death worldwide. Despite the global impact of $\mathrm{TB}$ and treatment received, a gap persists between treatment and quality of life of the patients especially in resource limited countries. This study therefore evaluated the health related quality of life of TB patients and the different factors influencing their quality of life at the post intensive phase of their treatment in the Fako division of the South-West Region of Cameroon. Methods: A hospital based cross sectional study was carried out in 4 tuberculosis treatment centers, namely two regional (Buea and Limbe) and two district (Tiko and Muyuka) hospitals in Fako Division between June and July 2017. One hundred and sixty-seven TB patients were enrolled in the study and relevant information from them was gathered using the SF-36 questionnaire. Various aspects such as their perception of the disease, their socio-demographics and socio-economics conditions were evaluated. Results: Of the 167 participants enrolled in the study, 95 (56.9\%) were male and 72 (43.1\%) were female. One hundred and thirty-three (79.6\%) of the 167 participants were affected by pulmonary tuberculosis and $34(20.4 \%)$ by extra-pulmonary tuberculosis. Fifty-five $(32.9 \%)$ were HIV positive and 90 (53.9\%) had been receiving treatment for 4 - 6 months, 73 (43.7\%) for $1-3$ months and $4(2.4 \%)$ for 7 - 9 months. The lowest and highest scores were recorded on the role limitation due to emotional problems scale (30.54 \pm
\end{abstract}


$35.36)$, and on the social functioning scale $(47.68 \pm 16.33)$ respectively. There was a significant difference $(\mathrm{p}=0.021)$, between pulmonary and extra pulmonary tuberculosis $(49.15 \pm 16.08$ vs. $41.91 \pm 16.25)$ on the social functioning. Financial difficulty restraining drug collection affected the general health perception $(p=0.003)$, vitality $(p=0.007)$, emotional well-being $(p<0.001)$ and social functioning ( $\mathrm{p}=0.05)$ of the patients. Low income affected the vitality $(p=0.039)$, emotional well-being $(p=0.015)$, role limitations due to physical $(\mathrm{p}=0.046)$ and emotional $(\mathrm{p}=0.003)$ problems of the patients. Equally, the HIV status affected their vitality $(\mathrm{p}<0.001)$ and emotional well-being ( $\mathrm{p}=0.011$ ). Conclusion: The quality of life of the TB patients in the study area was generally poor. Counselling of diagnosed TB patients and provision of financial assistance through a social package can improve the acceptance of the disease during their treatment period to avoid default and relapse.

\section{Keywords}

Tuberculosis, Quality of Life, Continuation Phase of Treatment, SF-36

Questionnaire, South West Region, Cameroon

\section{Introduction}

Tuberculosis (TB) infection compounded by antimicrobial resistance is the leading cause of death from a single infectious disease agent worldwide and a major contributor of death among people with Human Immunodeficiency Virus (HIV) [1]. In 2018, an estimated 10 million incident TB cases occurred with 1.5 million deaths [2]. Most of the people with TB live in low- and middle-income countries and the largest number of new TB cases occurred in the South-East Asian region, with $44 \%$ of new cases, followed by the African region, with $24 \%$ of new cases [3]. Mortality from TB is increasing in the developing world as the epidemic continues to grow. Particularly in Africa, crowded cities, poor and malnourished people with decreased immunity and inadequate healthcare services fuel the epidemic. Globally from 2000 to 2015, the incidence of TB (per 100,000 people) decreased from 172 to 142 per 100,000 people, in sub-saharan Africa from 344 to 276 and in Cameroon from 309 to 212 per 100,000 people. TB treatment has a positive impact on its prevalence and has averted 49 million deaths globally between 2000 and 2015 [4].

TB negatively impacts people and slowly destroys their body physically or psychologically. It is a chronic disease that leads to short or long-term disabilities. TB is treated for at least 6 months using a combination of drugs administered in two phases: the first two months of the intensive DOTS (Directly Observed Treatment short course) and the continuation phase [5]. As the symptoms of the disease manifest, the patients may suffer rejection or stigmatization which in turn lead to low self-esteem. Patient may equally lose their jobs or other means of livelihood and experience financial difficulties [6]. In Cameroon, TB 
treatment is free, consequently reducing the financial burden on the patient and facilitating their early recovery. TB patients who receive treatment often recover and do better than their counterparts who do not receive medication. However, some TB patients experience delayed or incomplete recovery which has been attributed to other challenges such as physical handicap related to the long term treatment as well as their perceptions about their disease. The combination of all these factors affects their healing process and may negatively impact their quality of life (QoL) [6].

There are few studies that have examined the QoL in patients with active TB in Africa and especially Cameroon, where the disease burden is high. Efforts to combat TB generally focus on disease management; from the prevention of mortality to the avoidance of morbidity, with little attention paid to the impact of the burden of illness and its therapy on the Health-Related Quality of Life (HRQoL) of TB patients [6] [7]. It is important to have an understanding of the disease based on all the predicted domains of QoL, including general health perceptions, somatic sensation, psychological health, spiritual well-being, social and role functioning especially in low income countries as ours. Evaluating the real impact of those factors might help to improve the management of TB patients and influence the life expectancy and their overall quality of life. Despite the availability of treatment, a gap persists between treatment and health status of TB patients. Several factors might adversely affect their healing and recovery process leading not only to poor adherence to treatment and subsequent development of drug resistance, but equally the perception of themselves, the family care (nuclear and extended), as well as the socio-economic and the socio-cultural environment. The purpose of this study was therefore to assess the HRQoL of TB patients in Fako Division, South West region of Cameroon.

\section{Patients and Methods}

\subsection{Study Area and Population}

This hospital-based, cross sectional study was carried out from June to July 2017 in 4 TB treatment centers located in Fako Division, one of the divisions in the South West Region of Cameroon with its capital at Limbe. The study included two regional (Buea and Limbe) and two district (Tiko and Muyuka) hospitals. The study population included TB patients, both men and women receiving free treatment at the hospital's DOTS centers. Only patients in the continuation phase of therapy and were 18 years old and above were included in the study. Patients still at the intensive phase of treatment were excluded considering their isolation and infectiousness.

\subsection{Ethical Considerations}

An ethical clearance was obtained from the Institutional Review Board of the Faculty of Health Sciences of the University of Buea and the administrative authorization from the Regional Delegation of the Ministry of Public Health for 
the South West Region and directors of the hospitals. Informed consent was equally obtained from each participant who had the opportunity to decline at any time from the study.

\subsection{Data Collection Tool}

The SF-36 questionnaire was used to determine HRQoL among the TB patients (https://www.rand.org/health-care/surveys tools/mos/36-item-short-form/surve y-instrument.html). The scale of the tool consists of 36 items with eight subscales: physical functioning (10 items), role limitations due to physical problems (4 items), bodily pain ( 2 items), general health perceptions ( 5 items), vitality (4 items), social functioning (2 items), role limitations due to emotional problems ( 3 items), and perceived mental health (5 items). In addition, the SF-36 has an item about health transition that is not part of any of the scales. Participant responses were coded, summed and transformed to a 0 - 100 scale, with higher scores indicating better physical and mental functioning, freedom from pain and overall better quality of life.

\subsection{Administration of Questionnaires}

Prior to the administration of the questionnaire, a pretesting was carried at the Military hospital of Buea which helped to correct lapses and errors to ensure quality and validity of the data collection tool [8] [9]. An one-day training equipped three selected graduates on how to administer the questionnaires to the study participants.

A SF-36 questionnaire form with a semi-structured questionnaire was administered to TB outpatients who agreed to participate in the study. The questionnaire captured data on socio-demographic characteristics such as the age, the gender, the marital status and educational level among others. Also, outcome variables like the perception of the disease, the relationship with family and community (whether the patient benefits from the family care and community's support or not), the physical aptitude and the economic capacity were captured to help to measure the patients' abilities to participate daily to social life activities and to estimate their overall quality of life.

\subsection{Sample Size, Sampling Technique and Procedure}

The sample size determination of this study was based on the Cochran's formula (https://www.statisticshowto.com/probability-and-statistics/find-sample-size/) assuming 95\% Confidence Interval and 5\% precision (Minimum of 156 participants obtained). Finally, 167 patients were included in the study. The records of the treatment health centers (Buea, Limbe, Tiko, Muyuka) were reviewed to obtain the demographic and treatment information of the patients. Using a simple random sampling technique, study participants were recruited from among $\mathrm{TB}$ patients who visited each treatment center to collect their monthly drugs. Only those who met the eligibility criteria were enrolled into the study. The phase of 
therapy and other information about the disease of each participant was further confirmed by personal interviews/self-evaluation. Questionnaires were administered to all enrolled participants to obtain relevant information.

\subsection{Data Management and Analysis}

Data obtained from the questionnaires were crosschecked to ensure that they were completely filled and devoid of errors. A code number was given to identify each participant. Data from the filled questionnaires were entered into a database (excel spread sheet) and were analyzed using the Statistical Package for the Social Sciences (SPSS), version 20.0. All p-values were two-tailed, and those less than 0.05 were considered statistically significant.

\section{Results}

\subsection{Distribution and Demography of Participants in the Studied Hospitals}

Of the 167 participants enrolled in this study, the majority [71 (42.5\%)] were from Limbe regional Hospital, followed by 51 (30.5\%) from the Buea regional hospital, 32 (19.2\%) from Muyuka district Hospital and 13 (7.8\%) from Tiko district hospital.

The demographic description of the participants presented in Table 1 shows that of the 167 participants enrolled in the study, 95 (56.9\%) were male and 72 (43.1\%) were female. The ages ranged from 19 to 68 years, with a mean age of $33.45 \pm 10.92$ years. Majority 79 (47.3\%) were aged below 29 years, while 17 (10.2\%) were above 50 years. Single people were the large proportion of the study population with $82(49.1 \%)$ participants. A total of $90(53.9 \%)$ attained secondary education while those with primary education were 58 (34.7\%). Fifty-seven (34.1\%) study participants were unemployed, 84 (62.7\%) had incomes ranging between 10,001 - 50,000 Fcfa (\$16.61 - \$83.07), 34 (25.4\%) earned below 10,000 Fcfa (\$16.61), 9 (6.7\%) earned between 50,001 - 100,000 Fcfa (\$83.07 $\$ 166.14)$ and 7 (5.2\%) earned above 100,001 Fcfa (\$166.14). The majority, 102 (61.1\%) of the 167 participants lived in urban areas and160 (95.8\%) were Christians.

One hundred and thirty-three (79.6\%) of the 167 participants were affected by pulmonary tuberculosis and $34(20.4 \%)$ by extra-pulmonary tuberculosis. Ninety (53.9\%) had been receiving treatment for 4 - 6 months, 73 (43.7\%) for 1 - 3 months and 4 (2.4\%) for 7 - 9 months. Of the 167 participants, 134 (80.2\%) were having the disease for the first time, 21 (12.6\%) were cases of relapse, $12(7.2 \%)$ were defaulters and there was no case of treatment failure. One hundred and fifty-one (90.4\%) of the 167 participants had no resistance to rifampicin or isoniazid, while $14(8.4 \%)$ had developed a resistance to either rifampicin or isoniazid. The majority [107 (64.1\%)] were HIV negative while 55 (32.9\%) were HIV positive. Seventeen (10.2\%) participants were smokers and 28 (16.8\%) attested to drinking alcohol regularly. 
Table 1. Socio-demographic characteristics of study participants.

\begin{tabular}{|c|c|c|c|}
\hline \multicolumn{2}{|r|}{ Characteristics } & \multirow{2}{*}{$\begin{array}{c}\begin{array}{c}\text { Frequency } \\
(\mathrm{n}=167)\end{array} \\
95\end{array}$} & \multirow{2}{*}{$\begin{array}{c}\text { Percentage } \\
(\%)\end{array}$} \\
\hline Gender & Male & & \\
\hline & Female & 72 & 43.1 \\
\hline \multirow[t]{5}{*}{ Age (years) } & Mean \pm SD & $33.45 \pm 10.92$ & \\
\hline & $<29$ & 79 & 47.3 \\
\hline & $30-39$ & 44 & 26.3 \\
\hline & $40-49$ & 27 & 16.2 \\
\hline & $\geq 50$ & 17 & 10.2 \\
\hline \multirow[t]{5}{*}{ Marital status } & Married & 57 & 34.1 \\
\hline & Single & 82 & 49.1 \\
\hline & Cohabitating & 19 & 11.4 \\
\hline & Divorced & 5 & 3.0 \\
\hline & Widowed & 4 & 2.4 \\
\hline \multirow[t]{3}{*}{ Level of education } & Primary & 58 & 34.7 \\
\hline & Secondary & 90 & 53.9 \\
\hline & Tertiary & 19 & 11.4 \\
\hline \multirow[t]{5}{*}{ Occupation } & Freelance & 50 & 29.9 \\
\hline & Housewife & 13 & 7.8 \\
\hline & Employee & 46 & 27.5 \\
\hline & Unemployed & 57 & 34.1 \\
\hline & Retired & 1 & 0.6 \\
\hline \multirow[t]{2}{*}{ Residence } & Urban & 102 & 61.1 \\
\hline & Rural & 65 & 38.9 \\
\hline \multirow[t]{4}{*}{ Income (Fcfa) } & $\leq 10,000(\$ 16.61)$ & 34 & 25.4 \\
\hline & $10,001-50,000(\$ 16.61$ - \$83.07), & 84 & 62.7 \\
\hline & $50,001-100,000(\$ 83.07-\$ 166.14)$ & 9 & 6.7 \\
\hline & $\geq 100,001(\$ 166.14)$ & 7 & 5.2 \\
\hline \multirow[t]{2}{*}{ Religion } & Christian & 160 & 95.8 \\
\hline & Muslim & 7 & 4.2 \\
\hline \multirow[t]{2}{*}{ TB type } & Pulmonary & 133 & 79.6 \\
\hline & Extra pulmonary & 34 & 20.4 \\
\hline \multirow[t]{3}{*}{ Duration (Months) } & $1-3$ & 73 & 43.7 \\
\hline & $4-6$ & 90 & 53.9 \\
\hline & $7-9$ & 4 & 2.4 \\
\hline \multirow[t]{3}{*}{ Disease status } & New case & 134 & 80.2 \\
\hline & Relapse & 21 & 12.6 \\
\hline & Default & 12 & 7.2 \\
\hline
\end{tabular}




\begin{tabular}{lccc} 
Continued & & & \\
\hline Drug resistance & Yes & 14 & 8.4 \\
& No & 151 & 90.4 \\
Don't know & 2 & 1.2 \\
HIV & Positive & 55 & 32.9 \\
& Negative & 107 & 64.1 \\
& Unknown & 5 & 3.0 \\
Smoke & Yes & 17 & 10.2 \\
& No & 150 & 89.8 \\
& Yes & 28 & 16.8 \\
Alcohol & No & 139 & 83.2 \\
\hline
\end{tabular}

\subsection{Description of Quality of Life' Domains of the Study Population}

\subsubsection{Overall Description of the Eight Domains of Quality of Life}

The scores of the participants' quality of life showed that the lowest was achieved on limitations due to emotional problems $(30.54 \pm 35.36)$ followed by physical functioning $(34.04 \pm 21.29)$ and physical health problems $(38.62 \pm 36.27)$. The highest score was recorded on the social functioning ( $47.68 \pm 16.33$ ), followed by the general health perception ( $43.65 \pm 17.07)$, the bodily pain $(41.59 \pm 25.35)$, the vitality $(40.90 \pm 16.01)$ and the emotional well-being (40.54 \pm 21.09$)$ (Table 2).

\subsubsection{Scores Comparison between Pulmonary and Extra-Pulmonary Tuberculosis}

The comparison between quality of life of TB (pulmonary and extra-pulmonary) showed that the overall mean of participants for physical functioning was 34.73 \pm 20.12 , with no significant difference $(\mathrm{p}=0.574)$ between extra-pulmonary (35.88 \pm 18.19$)$ and pulmonary TB patients $(33.57 \pm 22.05)$. Extra pulmonary patients were more limited in their roles due to physical health $(41.91 \pm 33.55)$ and emotional problems $(31.37 \pm 25.87)$ than those with pulmonary TB under the same condition with respectively $37.78 \pm 37$ and $30.33 \pm 37.48$ means ( $\mathrm{p}=$ $0.555 \mathrm{vs} \mathrm{p}=0.878$ ). The mean in vitality (energy and fatigue) was $41.82 \pm 16.65$ for all the participants, with a mean score in those with extra-pulmonary TB of $43.38 \pm 17.74$ against $40.26 \pm 15.55$ for pulmonary TB $(\mathrm{p}=0.312)$. Participants with extra-pulmonary TB $(44.26 \pm 21.25)$ felt better emotionally though not significantly $(\mathrm{p}=0.249)$ than those with pulmonary TB $(39.59 \pm 21.01)$. Participants with extra-pulmonary TB $(48.60 \pm 23.69)$ felt no significant bodily pain ( $p$ $=0.070)$ than those who suffered from pulmonary TB $(39.79 \pm 25.53)$. The mean in general health was better $(\mathrm{p}=0.073)$ in participants with pulmonary TB $(44.85 \pm 17.64)$ than those with extra-pulmonary TB $(38.97 \pm 13.86)$. Equally, the domain with significant difference $(\mathrm{p}=0.021$ ) was between the social functioning and the type of $\mathrm{TB}$, where the mean of participants with pulmonary $\mathrm{TB}$ was $49.15 \pm 16.08$ and those with extra-pulmonary TB was $41.91 \pm 16.25$ (Table 3). 
Table 2. Scores of the quality of life domains of the study participants.

\begin{tabular}{cc}
\hline SF-36 categories & Scores \\
\hline Physical functioning & $34.04 \pm 21.29$ \\
Limitations due to physical health problems & $38.62 \pm 36.27$ \\
Limitations due to emotional problems & $30.54 \pm 35.36$ \\
Vitality (Energy/fatigue) & $40.90 \pm 16.01$ \\
Emotional well-being & $40.54 \pm 21.09$ \\
Social functioning & $47.68 \pm 16.33$ \\
Bodily pain & $41.59 \pm 25.35$ \\
General health perception & $43.65 \pm 17.07$ \\
\hline
\end{tabular}

Table 3. Comparison of eight SF-36 category scores between pulmonary and extra-pulmonary TB patients.

\begin{tabular}{ccccc}
\hline \multirow{2}{*}{ SF-36 categories } & \multicolumn{3}{c}{ Scores } & \\
\cline { 2 - 5 } & $\begin{array}{c}\text { Pulmonary } \\
\text { TB }(\mathrm{n}=133)\end{array}$ & $\begin{array}{c}\text { Extra-Pulmonary } \\
\mathrm{TB}(\mathrm{n}=34)\end{array}$ & Overall & \\
\hline Physical functioning & $33.57 \pm 22.05$ & $35.88 \pm 18.19$ & $34.73 \pm 20.12$ & 0.574 \\
Limitations due to physical health & $37.78 \pm 37$ & $41.91 \pm 33.55$ & $39.85 \pm 35.28$ & 0.555 \\
Limitations due to emotional problems & $30.33 \pm 37.48$ & $31.37 \pm 25.87$ & $30.85 \pm 31.68$ & 0.878 \\
Vitality (Energy/fatigue) & $40.26 \pm 15.55$ & $43.38 \pm 17.74$ & $41.82 \pm 16.65$ & 0.312 \\
Emotional well-being & $39.59 \pm 21.01$ & $44.26 \pm 21.25$ & $41.93 \pm 21.13$ & 0.249 \\
Social functioning & $49.15 \pm 16.08$ & $41.91 \pm 16.25$ & $45.53 \pm 16.17$ & 0.021 \\
Bodily pain & $39.79 \pm 25.53$ & $48.60 \pm 23.69$ & $44.20 \pm 24.61$ & 0.070 \\
General health perception & $44.85 \pm 17.64$ & $38.97 \pm 13.86$ & $41.91 \pm 15.75$ & 0.073 \\
\hline
\end{tabular}

\subsection{Patients' Perception about the Disease and Health Care Services}

Of the 167 participants, 68 (40.7\%) accepted their disease condition as TB patients, mostly 44 (26.3\%), completely $24(14.4 \%)$ and $32(19.2 \%)$ accepted it moderately. However, 23 participants (13.8\%) and 44 (26.3\%) did not or did little accept their disease respectively. On the other hand, Figure 1 equally shows that of the 167 participants, $69(41.3 \%)$ and 18 (10.8\%) were satisfied or very satisfied with the health care services provided respectively, with only $18(10.8 \%)$ very dissatisfied, 14 (8.4\%) dissatisfied and 48 (28.7\%) neither satisfied nor dissatisfied.

\subsection{Perception about the Fear of Relapse and Optimism to Be Cured}

The fear of relapse was present in about half of the study participants [82/167 (49.1\%)], although 36 (21.5\%) of the 167 respondents never expressed such fear. The desire to be cured was expressed by the majority of the 167 participants, 91 (54.5\%) always optimistic, $52(31.1 \%)$ very often and $20(12.0 \%)$ were quite often. Only 4 (2.4\%) were pessimistic (Figure 2 ). 

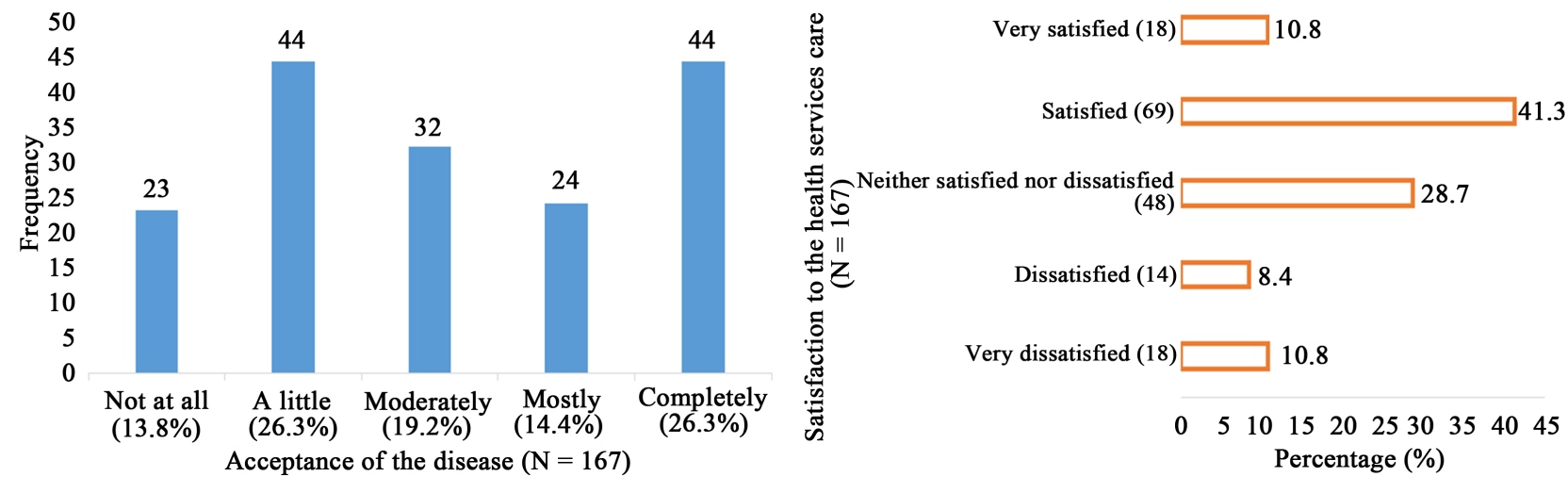

Figure 1. Disease acceptance and health care services satisfaction by respondents.

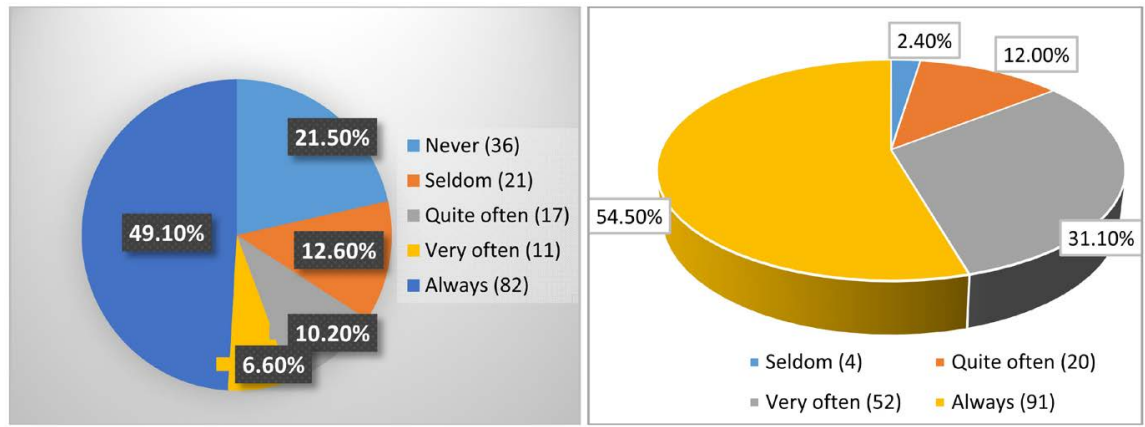

Figure 2. Participants' fear of disease relapse and optimism to be cured.

\subsection{Factors Associated with Quality of Life}

\subsubsection{Quality of LIFE and Socio-Demographic Factors}

The comparative analysis of the quality of life of the 167 participants and socio-demographic factors is shown in Table 4. Physical functioning was significantly affected by age $(p=0.019)$, marital status $(p<0.001)$ and those still working during their treatment $(\mathrm{p}=0.004)$. Participants above 40 years had a better physical functioning $(40.45 \pm 15.66)$ than those age below 40 years old $(31.75 \pm 22.59)$. Those who had experienced a marital life before (separated, divorced or widowed) had a better physical functioning ( $45.56 \pm 8.82$ ), followed by the married $(37.11 \pm 18.85)$, the single $(35.61 \pm 21.49)$ and those who cohabitated $(12.63 \pm 19.25)$. On the other hand, the mean of the physical functioning of those who had stop working during their treatment was higher $(37.82 \pm 19.49)$ compared to those who were still working during their treatment $(28.26 \pm$ 22.74).

Limitations due to physical health problems was influenced by marital status ( $\mathrm{p}<0.001)$ with a mean of $71.05 \pm 35.61$ for participants who cohabitated, followed by those who were single (37.50 \pm 33.39$)$, married (32.02 \pm 36.52$)$ or were married before $(22.22 \pm 26.35)$. There was a significant difference between the limitations due to physical problems and the duration of treatment $(\mathrm{p}=0.005)$. Those who were on treatment for a period of $4-6$ months were more limited in their role $(46.94 \pm 37.07)$ than those on treatment for a period of $1-3$ months $(29.11 \pm 32.28)$ or 7 - 9 months $(25.00 \pm 50.00)$. 
Table 4. Comparison of the eight domains of quality of life and the socio-demographic factors.

\begin{tabular}{|c|c|c|c|c|c|c|c|c|}
\hline & $\begin{array}{l}\text { Physical } \\
\text { functioning }\end{array}$ & $\begin{array}{l}\text { Role limitations } \\
\text { due to physical } \\
\text { problems }\end{array}$ & $\begin{array}{l}\text { Role limitations } \\
\text { due to emotional } \\
\text { problems }\end{array}$ & $\begin{array}{c}\text { Vitality } \\
\text { (energy } \\
\text { and fatigue) }\end{array}$ & $\begin{array}{l}\text { Emotional } \\
\text { well-being }\end{array}$ & $\begin{array}{c}\text { Social } \\
\text { functioning }\end{array}$ & Bodily pain & $\begin{array}{l}\text { General } \\
\text { health } \\
\text { perception }\end{array}$ \\
\hline Gender & $\mathrm{P}=0.417$ & $\mathrm{P}=0.112$ & $\mathrm{P}=0.055$ & $\mathrm{P}=0.494$ & $P=0.466$ & $\mathrm{P}=0.380$ & $\mathrm{P}=0.047$ & $\mathrm{P}=0.978$ \\
\hline Male & $35.21 \pm 21.06$ & $34.74 \pm 34.46$ & $25.96 \pm 31.95$ & $40.15 \pm 17.24$ & $41.58 \pm 21.39$ & $46.71 \pm 17.60$ & $44.97 \pm 23.95$ & $43.68 \pm 15.83$ \\
\hline Female & $32.50 \pm 21.64$ & $43.75 \pm 38.17$ & $36.57 \pm 38.82$ & $41.87 \pm 14.30$ & $39.17 \pm 20.74$ & $48.96 \pm 14.50$ & $37.12 \pm 26.60$ & $43.61 \pm 18.69$ \\
\hline Age group & $\mathrm{P}=0.019$ & $\mathrm{P}=0.400$ & $\mathrm{P}=0.109$ & $P=0.619$ & $\mathrm{P}=0.991$ & $\mathrm{P}=0.435$ & $\mathrm{P}=0.093$ & $P=0.764$ \\
\hline$<40$ years & $31.75 \pm 22.59$ & $40.04 \pm 36.04$ & $27.91 \pm 33.71$ & $40.53 \pm 15.81$ & $40.53 \pm 21.10$ & $48.27 \pm 16.96$ & $43.56 \pm 26.08$ & $43.41 \pm 16.73$ \\
\hline$\geq 40$ years & $40.45 \pm 15.66$ & $34.66 \pm 37.05$ & $37.88 \pm 39.11$ & $41.93 \pm 16.71$ & $40.57 \pm 21.30$ & $46.02 \pm 14.46$ & $36.08 \pm 22.56$ & $44.32 \pm 18.16$ \\
\hline Marital status & $\mathrm{P}<0.001$ & $\mathrm{P}<0.001$ & $\mathrm{P}=0.143$ & $\mathrm{P}=0.098$ & $\mathrm{P}=0.022$ & $\mathrm{P}=0.388$ & $\mathrm{P}=0.028$ & $\mathrm{P}=0.057$ \\
\hline Married & $37.11 \pm 18.85$ & $32.02 \pm 36.52$ & $36.84 \pm 38.67$ & $37.02 \pm 16.06$ & $35.26 \pm 20.56$ & $50.22 \pm 14.46$ & $37.85 \pm 26.22$ & $40.44 \pm 18.23$ \\
\hline Single & $35.61 \pm 21.49$ & $37.50 \pm 33.39$ & $30.49 \pm 35.21$ & $41.95 \pm 15.67$ & $40.91 \pm 20.49$ & $46.49 \pm 18.45$ & $46.22 \pm 24.37$ & $43.48 \pm 16.34$ \\
\hline Cohabitating & $12.63 \pm 19.25$ & $71.05 \pm 35.61$ & $17.54 \pm 28.04$ & $45.26 \pm 18.06$ & $50.53 \pm 22.72$ & $48.03 \pm 12.68$ & $29.47 \pm 23.43$ & $48.95 \pm 15.60$ \\
\hline Married before & $45.56 \pm 8.82$ & $22.22 \pm 26.35$ & $18.52 \pm 17.57$ & $46.67 \pm 9.68$ & $49.44 \pm 18.10$ & $41.67 \pm 12.50$ & $48.61 \pm 23.42$ & $54.44 \pm 14.02$ \\
\hline Level of education & $\mathrm{P}=0.078$ & $P=0.062$ & $\mathrm{P}=0.011$ & $P=0.03$ & $\mathrm{P}<0.001$ & $P=0.359$ & $\mathrm{P}=0.270$ & $P<0.001$ \\
\hline Primary & $37.41 \pm 20.86$ & $32.76 \pm 36.33$ & $21.84 \pm 30.94$ & $43.79 \pm 13.77$ & $45.95 \pm 16.56$ & $46.55 \pm 17.65$ & $39.01 \pm 20.30$ & $49.31 \pm 11.79$ \\
\hline Secondary & $33.83 \pm 19.04$ & $38.89 \pm 34.57$ & $32.22 \pm 36.87$ & $40.78 \pm 16.51$ & $40.56 \pm 23.22$ & $47.36 \pm 15.73$ & $41.50 \pm 27.36$ & $42.83 \pm 18.30$ \\
\hline Tertiary & $24.74 \pm 29.70$ & $55.26 \pm 40.47$ & $49.12 \pm 34.01$ & $32.63 \pm 17.82$ & $23.95 \pm 13.50$ & $52.63 \pm 14.78$ & $49.87 \pm 28.75$ & $30.26 \pm 17.20$ \\
\hline $\begin{array}{c}\text { Duration of } \\
\text { treatment }\end{array}$ & $P=0.115$ & $\mathrm{P}=0.005$ & $\mathrm{P}=0.064$ & $P=0.730$ & $P=0.452$ & $P=0.625$ & $P=0.473$ & $P=0.989$ \\
\hline $1-3$ months & $37.60 \pm 20.31$ & $29.11 \pm 32.28$ & $23.29 \pm 30.27$ & $41.44 \pm 13.29$ & $39.79 \pm 18.02$ & $48.97 \pm 18.61$ & $42.74 \pm 24.30$ & $43.42 \pm 16.22$ \\
\hline 4- 6 months & $30.89 \pm 21.56$ & $46.94 \pm 37.07$ & $36.30 \pm 37.94$ & $36.30 \pm 37.34$ & $41.67 \pm 23.52$ & $46.81 \pm 14.58$ & $41.31 \pm 26.45$ & $43.83 \pm 17.83$ \\
\hline 7 - 9 months & $40.00 \pm 34.04$ & $25.00 \pm 50.00$ & $33.33 \pm 47.14$ & $33.33 \pm 47.14$ & $28.75 \pm 12.50$ & $43.75 \pm 7.22$ & $26.88 \pm 17.37$ & $43.75 \pm 19.31$ \\
\hline Adherence & $\mathrm{P}=0.837$ & $\mathrm{P}=0.332$ & $\mathrm{P}=0.006$ & $\mathrm{P}=0.041$ & $P=0.001$ & $P=0.077$ & $P=0.237$ & $P=0.001$ \\
\hline Yes & $33.89 \pm 21.60$ & $39.82 \pm 36.35$ & $33.81 \pm 36.86$ & $39.79 \pm 16.95$ & $38.14 \pm 21.79$ & $48.66 \pm 16.61$ & $42.61 \pm 26.47$ & $41.75 \pm 17.72$ \\
\hline No & $34.81 \pm 19.98$ & $32.41 \pm 35.91$ & $13.58 \pm 19.08$ & $46.67 \pm 7.72$ & $52.96 \pm 10.40$ & $42.59 \pm 13.98$ & $36.30 \pm 17.97$ & $53.52 \pm 7.82$ \\
\hline HIV status & $\mathrm{P}=0.832$ & $\mathrm{P}=0.897$ & $\mathrm{P}=0.442$ & $\mathrm{P}<0.001$ & $\mathrm{P}=0.011$ & $P=0.177$ & $\mathrm{P}=0.027$ & $\mathrm{P}=0.001$ \\
\hline Positive & $34.45 \pm 23.07$ & $39.10 \pm 34.95$ & $26.67 \pm 35.37$ & $48.09 \pm 12.86$ & $46.64 \pm 16.05$ & $45.68 \pm 13.22$ & $35.95 \pm 26.97$ & $50.00 \pm 15.93$ \\
\hline Negative & $33.69 \pm 20.82$ & $38.32 \pm 36.39$ & $31.15 \pm 34.95$ & $36.54 \pm 15.97$ & $37.76 \pm 22.92$ & $49.30 \pm 17.33$ & $45.21 \pm 24.00$ & $40.56 \pm 16.10$ \\
\hline Smoke & $P=0.129$ & $P=0.691$ & $\mathrm{P}=0.113$ & $\mathrm{P}=0.471$ & $P=0.002$ & $\mathrm{P}=0.054$ & $P=0.526$ & $\mathrm{P}=0.215$ \\
\hline Yes & $41.47 \pm 10.57$ & $35.29 \pm 21.76$ & $17.65 \pm 23.91$ & $38.24 \pm 14.68$ & $55.59 \pm 19.75$ & $40.44 \pm 15.01$ & $45.30 \pm 12.34$ & $48.53 \pm 19.26$ \\
\hline No & $33.20 \pm 22.05$ & $39.00 \pm 37.60$ & $32.00 \pm 36.20$ & $41.20 \pm 16.18$ & $38.83 \pm 20.61$ & $48.50 \pm 16.32$ & $41.17 \pm 26.42$ & $43.10 \pm 16.79$ \\
\hline Alcohol & $\mathrm{P}=0.323$ & $\mathrm{P}=0.302$ & $\mathrm{P}=0.136$ & $\mathrm{P}=0.521$ & $\mathrm{P}=0.221$ & $P=0.658$ & $P=0.002$ & $P=0.606$ \\
\hline Yes & $37.68 \pm 23.43$ & $32.14 \pm 31.81$ & $21.43 \pm 34.20$ & $42.68 \pm 19.41$ & $45.00 \pm 23.33$ & $46.43 \pm 15.16$ & $28.21 \pm 23.61$ & $45.18 \pm 23.75$ \\
\hline No & $33.31 \pm 20.85$ & $39.93 \pm 37.07$ & $32.37 \pm 35.43$ & $40.54 \pm 15.30$ & $39.64 \pm 20.58$ & $47.93 \pm 16.59$ & $44.28 \pm 24.91$ & $43.35 \pm 15.48$ \\
\hline Still working & $P=0.004$ & $P=0.080$ & $\mathrm{P}=0.158$ & $\mathrm{P}=0.141$ & $P=0.229$ & $P=0.608$ & $\mathrm{P}<0.001$ & $P=0.882$ \\
\hline Yes & $28.26 \pm 22.74$ & $44.70 \pm 34.11$ & $25.76 \pm 34.49$ & $38.64 \pm 14.87$ & $38.11 \pm 20.92$ & $48.48 \pm 15.58$ & $51.02 \pm 27.65$ & $43.41 \pm 16.20$ \\
\hline No & $37.82 \pm 19.49$ & $34.65 \pm 37.24$ & $33.66 \pm 35.74$ & $42.38 \pm 16.62$ & $42.13 \pm 21.15$ & $47.15 \pm 16.85$ & $35.42 \pm 21.74$ & $43.81 \pm 17.69$ \\
\hline
\end{tabular}


However, limitations due to emotional problems was instead affected by the level of education $(\mathrm{p}=0.011)$ and adherence $(\mathrm{p}=0.006)$. Participants with tertiary education $(49.12 \pm 34.01)$ were more limited than those with secondary $(32.22 \pm 36.87)$ and primary $(21.84 \pm 30.94)$ education. Those who were adherent were more limited $(33.81 \pm 36.86)$ in their role due to emotional problems than those who were not adherent $(13.58 \pm 19.08)$.

Vitality and emotional well-being shared respectively the influence of level of education ( $p=0.03$ vs. $p<0.001$ ), adherence ( $p=0.041$ vs. $p=0.001$ ) and HIV status ( $\mathrm{p}<0.001$ vs. $\mathrm{p}=0.011)$. Participants with primary level of education $(43.79 \pm 13.77)$ had more energy and vitality than those with secondary (40.78 \pm $16.51)$ and tertiary $(32.63 \pm 17.82)$ level of education. Participants who were not adherent $(46.67 \pm 7.72)$ had a better energy and felt less tired than those who were adherent ( $39.79 \pm 16.95)$. Those who were HIV positive (48.09 \pm 12.86$)$ had a better vitality than HIV negative $(36.54 \pm 15.97)$ participants.

Participants who cohabitated $(50.53 \pm 22.72)$ and those who experienced marital life before $(49.44 \pm 18.10)$ had a better emotional well-being $(\mathrm{p}=0.022)$ than the single ( $40.91 \pm 20.49)$ or married ones $(35.26 \pm 20.56)$. Participants with primary level of education $(45.95 \pm 16.56)$ had a better emotional well-being than those with secondary $(40.56 \pm 23.22)$ and tertiary $(23.95 \pm 13.50)$ level of education. Those who were not adherent had a significant emotional well-being $(52.96 \pm 10.40)$ than those who were adherent $(38.14 \pm 21.79)$. HIV positive patients (46.64 \pm 16.05$)$ had a significant emotional well-being than HIV negative ones $(37.76 \pm 22.92)$. Smokers $(55.59 \pm 19.75)$ also had significant well-being compared to the non-smokers $(38.83 \pm 20.61)$. Gender was significantly associated with bodily pain $(\mathrm{p}=0.047)$, with female $(37.12 \pm 26.60)$ more affected than male $(44.97 \pm 22.95)$. Moreover, participants who were married (37.85 \pm $26.22)$ and those who cohabitated $(29.47 \pm 23.43)$ had significantly more bodily pain $(\mathrm{p}=0.028)$ than those who experienced marital life before $(48.61 \pm 23.42)$ and those who were single $(46.22 \pm 24.37)$. HIV positive participants $(35.95 \pm$ $26.97)$ felt more pain $(\mathrm{p}=0.027)$ than HIV negative ones $(45.21 \pm 24.00)$. Those who used alcohol $(28.21 \pm 23.61)$ had significantly more bodily pain $(\mathrm{p}=0.002)$ than those who did not use alcohol $(44.28 \pm 24.91)$, and those who stopped working $(35.42 \pm 21.74)$ felt more bodily pain than those who were still working $(51.02 \pm 27.65)$ during their treatment $(\mathrm{p}<0.001)$.

Social functioning was not affected by any factor, while the general health perception on the other hand was associated with level of education $(\mathrm{p}<0.001)$, adherence $(\mathrm{p}=0.001)$ and HIV status $(\mathrm{p}=0.001)$. Participants with primary level of education $(49.31 \pm 11.79)$ had a better general health perception than those who had attended secondary $(42.83 \pm 18.30)$ and tertiary $(30.26 \pm 17.20)$ education. Participants who did not adhere $(53.52 \pm 7.82)$ had a better general health perception than those who adhered $(41.75 \pm 17.72)$. Those who were HIV positive $(50.00 \pm 15.93)$ had a better general health perception than those who were HIV negative (41.56 \pm 16.10$)$. 


\subsubsection{Quality of Life and Socio-Economic Factors}

Financial difficulty and drug collection affected domains such as vitality $(\mathrm{p}=$ 0.007), emotional well-being ( $p<0.001)$ social functioning $(p=0.05)$ and general health perception $(p=0.0003)$.The response related to enough money to meet their needs affected the role limitations due to physical $(\mathrm{p}=0.046)$ and emotional $(\mathrm{p}=0.003)$ problems, as well as vitality $(\mathrm{p}=0.039)$ and emotional well-being $(\mathrm{p}=0.015)($ Table 5).

\section{Discussion}

There is paucity of information on the quality of life of patients with pulmonary TB in Cameroon. Considering that active TB disease exerts a substantial toll on QoL ranging from somatic symptoms related to disease and treatment to psychological distress from social isolation and stigmatization [9] [10] [11], this study therefore assessed the factors influencing the QoL as well as the perception of the participants about their disease conditions, and the predictors of low QoL scores of tuberculosis patients who were on the continuation phase (at least two months) of their treatment in the Fako Division of the South West Region of Cameroon. The health status was measured for the 8 following domains: physical functioning, role limitations due to physical and emotional problems, vitality (energy and fatigue), emotional well-being, social functioning, pain and general health perception.

The number of male (56.9\%) and female (43.1\%) in this study is comparable to previous findings reporting a higher prevalence of tuberculosis among men than women [11] [12] [13] [14]. Our study showed that women had lower scores to bodily pain compared to male contrary to a study where women's scores were significantly higher than men's on pain intensity, pain anxiety, and current functioning [15]. This may not be unconnected to the fact that the study setting and population were different, even though another study showed that both sexes fared comparably in the physical domains [16]. Rajeswari et al. using the SF 36 instrument of quality of life evaluation in a south Indian population had

Table 5. Comparison of the eight domains of quality of life and the socio-economic factors.

\begin{tabular}{cccccccccc}
\hline & $\begin{array}{c}\text { Physical } \\
\text { functioning }\end{array}$ & $\begin{array}{c}\text { Role limitations } \\
\text { due to physical } \\
\text { problems }\end{array}$ & $\begin{array}{c}\text { Role limitations } \\
\text { due to emotional } \\
\text { problems }\end{array}$ & $\begin{array}{c}\text { Vitality } \\
\text { (Energy and } \\
\text { fatigue })\end{array}$ & $\begin{array}{c}\text { Emotional } \\
\text { well-being }\end{array}$ & $\begin{array}{c}\text { Social } \\
\text { functioning }\end{array}$ & $\begin{array}{c}\text { Bodily pain } \\
\text { health } \\
\text { perception }\end{array}$ \\
\hline $\begin{array}{c}\text { Financial } \\
\text { difficulty/Drug } \\
\text { collection }\end{array}$ & $\mathrm{P}=0.336$ & $\mathrm{P}=0.851$ & $\mathrm{P}=0.452$ & $\mathrm{P}=0.007$ & $\mathrm{P}<0.001$ & $\mathrm{P}=0.050$ & $\mathrm{P}=0.775$ & $\mathrm{P}=0.003$ \\
Yes & $36.17 \pm 20.94$ & $37.92 \pm 36.09$ & $27.78 \pm 29.54$ & $45.33 \pm 13.77$ & $50.50 \pm 17.31$ & $44.38 \pm 15.51$ & $40.83 \pm 22.75$ & $48.83 \pm 9.89$ \\
No & $32.85 \pm 21.49$ & $39.02 \pm 36.53$ & $32.09 \pm 38.29$ & $38.41 \pm 16.69$ & $34.95 \pm 21.02$ & $49.53 \pm 16.55$ & $42.00 \pm 26.79$ & $40.75 \pm 19.45$ \\
$\begin{array}{c}\text { Enough money } \\
\text { for needs }\end{array}$ & $\mathrm{P}=0.138$ & $\mathrm{P}=0.046$ & $\mathrm{P}=0.003$ & $\mathrm{P}=0.039$ & $\mathrm{P}=0.015$ & $\mathrm{P}=0.065$ & $\mathrm{P}=0.070$ & 0.152 \\
Yes & $30.94 \pm 21.66$ & $45.70 \pm 35.23$ & $40.63 \pm 37.78$ & $37.66 \pm 15.91$ & $35.55 \pm 21.42$ & $44.73 \pm 15.07$ & $46.03 \pm 27.19$ & $41.25 \pm 18.30$ \\
No & $35.97 \pm 20.93$ & $34.22 \pm 36.38$ & $24.27 \pm 32.40$ & $42.91 \pm 15.82$ & $43.64 \pm 20.37$ & $49.51 \pm 16.87$ & $38.79 \pm 23.84$ & $45.15 \pm 16.17$ \\
\hline
\end{tabular}


reported lower scores in females compared with males in the mental and social domains in contrast to our present findings [17]. This might result from socio-cultural differences between the populations studied.

As recorded in several studies [16] [17] [18], the means of the various categories of the quality of life in our study was worse with most scores under 50. A study has shown that the scores obtained in all the domains were lower in the pulmonary patients group compared to the control group [13], while another study reported that the overall physical and mental health scores were 42.5 and 40.7 respectively. Emotional role, general health and bodily pain had the lowest sub-scale scores, while mental health, energy and fatigue had the highest domain scores [14].

From our results, patients with pulmonary TB had the highest score on the social functioning (47.68) and recorded the lowest score on the role limitation (30.54) probably due to emotional problems. Similar findings were reported in a study carried out in Iran [8] using the SF-36 form which revealed a lowest score in role limitations due to emotional problems but a highest score in general health perception. The role limitation may probably be due to the fact that most patients had difficulty to accept their medical condition and consequently had a lower self-perception/esteem of themselves, or because they had physical pain and were limited to achieve many normal tasks than they were previously used to. A study carried out in India using WHO QOL BREF has also shown that the worst domains affected by the disease were physical domain followed by the psychological domain that impaired the patients' ability to carry on normal physical activities [19]. A study has also shown that health-related QOL declines in TB patients with the physical scales being the most affected [20], as shown in our study where physical activity affected their ability to work.

Mojgan et al. in their study at different phases (before, at initial and at end) of treatment showed that the lowest score in TB patients was related to physical functioning and vitality after two months of treatment with no significant difference between two and six months after treatment [18]. Although our study did not compare the intensive against the continuation phase of treatment, but focused only at the latter phase, quantitative studies have reported a wide range of values for the health impairment associated with TB before, during, and after treatment [21]. A study carried out in India on similar patients reported that although the initial health status was less than $7 \%$ at the onset of the disease, it significantly improved during and after the treatment (more than 78\%) [22], indicating that the lower score in our study may have resulted from the fact that psychological outcome of the disease during treatment may probably be due to stigmatization from the community, consequently leading to depression among patients.

The acceptance rate shows that more than half of the participants (59.3\%) in our study had difficulty in accepting their medical condition. Some studies reported that the patients' reaction when they were told about their disease was 
worry (50\%) or suicidal thoughts (9\%) [6] [22]. Freitas et al. in their Brazilian study on knowledge and perception of TB patients under DOTS revealed that $50 \%$ of them felt ashamed of having TB [23]. Those similarities in the perception of the disease by patients can be explained by the fact that TB is considered a shameful disease leading to isolation of the patients in order to avoid transmission, and thus the apprehension of being rejected or discriminated. Conversely, some participants in Marra et al.' study stated that acquiring TB had resulted in positive health behaviour modification. Many participants took the development of TB to be a "wake-up" call to change their lifestyle and improve their unhealth lifestyles by either eliminating or reducing drug and alcohol intake, increasing exercise, or eating better. These findings were also reported by the Baltimore study group suggesting that the positive health behaviour impacts of this disease might be widespread among those afflicted with TB in North America, with an overall significant effect on health behaviour modification, especially that the patients were engaged in other high-risk behaviours such as use of illicit drugs [12].

In our study, the fear of relapse appears to be always present in about half of the study participants, and some had the same feeling even if it was less often. Raneswarj et al. reported that only $54 \%$ of patients perceived "happy mental status" at the end of treatment and there was no change in social stigma in both men and women [24], even if it is not well known how emotional health changes with treatment [15]. This similarity could be explained by the fact that participants perceived their limitations in both physical and mental domains and consequently an impaired QoL. This resulted in the limitation of their social activities like going to church or visit their friends and relatives. The disease equally affected their economic status as for instance agricultural activities, where strength and physical capacities are required to work in farms [6]. Despite about half of our study participants expressing the permanent feeling of fear of having the disease again, $86 \%$ were optimistic to be cured, as shown in a Nigerian study [6]. Another aspect of the patients' perception was that $52 \%$ of the participants were satisfied or very satisfied with the health care services provided in the various hospitals while a study carried out in Canada revealed negative feelings associated with TB diagnosis and the initial hospitalization amongst their participants. Some participants expressed frustration with their primary care physicians for inappropriate management related to diagnosis due to lack of provider knowledge with respect to TB [12]. Many participants in the same study either felt that they had a delayed diagnosis or delayed treatment due to issues related to their health care provider [12], unlike our study where the close proximity an empathy of health care provider in our setting might explain the positive feeling of our participants. Patients are welcome in the TB unit where they receive counselling speech on the curable aspect of the disease, drug intake schedule and are reminded of their appointment which is very important to avoid developing a resistance to drugs or defaulting. 
Patients who were at the 4 - 6 months of treatment were more limited in their activities due to physical problem probably because the healing process was still on-going and the recovery incomplete. Some differences in physical functioning and bodily pain were found among the gender, the marital status, the HIV status and those taking alcohol. In our study, the participants with primary level of education, those who were not drug adherent and those HIV positive showed significantly better general health perception. A study has found that general health perceptions affected divorced patients more significantly than single and married patients [8]. Equally, participants who were not adherent and those with primary level of education may not really understand the implications associated to their medical condition probably due to their lack of disease knowledge.

TB and HIV co-infection has been reported by Louw et al. to be a factor that significantly impairs the participants' quality of life and weaken their physical and mental functioning [14]. This result was similar to our study probably because the participants who are HIV positive are counselled at two different units of the hospital (TB and HIV units), which help them to be stronger psychologically, to have a good mental to accept their medical condition, though their immune system remains weak.

We found as another study that there were significant correlations between those who had financial difficulty and QoL [25]. Their medical condition may result in job loss because diagnosed patients are less likely to find job, and less able to work and care for their families, thereby creating a greatest financial burden on the poor [15]. Participants who had difficulty collecting their drugs on time and those who did not have enough money to meet their need had more vitality and emotional well-being than those who did not have financial difficulty. The fact that they are limited financially and that they are sick can be a catalyst for them to make more efforts to find money and means to treat themselves while those who have enough money are not preoccupied by financial issues, but remain lethargic and more concerned by their health status. Marra et al. reported a different finding that no participants complained about a job loss because of TB. One possible reason for this observation could be due to low rate of employment in their sample with only $26 \%$ being full or part-time employees [12].

On the other hand, and contrary to the above perception, some differences in emotional well-being, vitality, social functioning and general health perception were found among patients with financial difficulties. A study in the United States demonstrated that HRQOL among pulmonary TB patients in a socioeconomically depressed setting was impaired compared with the general population [11]. The lack of money may equally affect some patients emotionally. Our study shows that participants with low socioeconomic status had a substantial correlation with HRQOL, because they had a low perception of their health [10]. It could be assumed that before being diagnosed with the disease, they were already facing financial difficulties which were further compounded by their present medical condition. 


\section{Limitation}

This study was a cross-sectional study by nature and inference about the causal relationship was not possible. This study is mainly a subjective study whose reliability is based on participants' sincerity who may not always give exact answers. Therefore, there is a risk of bias related to participants.

\section{Conclusion}

This study showed that TB patients had a general low quality of life, with the highest score on social functioning and the lowest in limitation probably as a result of emotional problem or economic status, as shown by their financial difficulty and lack of money to meet their need. Most of the participants accepted their health condition and were always afraid of relapsing. Males suffered from bodily pain more than females. Gender, level of education, adherence, HIV status, alcohol and financial difficulties were the main factors affecting the quality of life in TB patients in the study area. It will be important for patients to consider TB as a common disease which can be treated. Creating a support group to share their experiences and challenges as well as a psychological cell in DOTS center might help to improve their acceptance of the disease. Family members should be encouraged to support their patient relatives through counselling and sensitization among the population to avoid stigmatization and discrimination of TB patients. Provision of a social package to assist patients during and after the treatment period will go a long way to improve their general condition.

\section{Acknowledgements}

The authors are grateful to all the TB patients who agreed to participate in this study. We equally thank the directors and staff of the hospitals concerned who cooperated in providing the relevant information used in this study.

\section{Conflicts of Interest}

The authors declared no conflict of interest.

\section{Authors Contributions}

Conceptualization: BDTP, PF, TNN, PWG. Formal analysis: BDTP, PWG. Investigation: BDTP, PWD. Methodology: TNN, PWG, AEA. Validation: BDTP, PF.AEA. Visualization: PF, TNN. Writing original draft: BDTP, PWG. Writing review \& editing: BDTP, PF, TNN, PWG, AEA.

\section{References}

[1] World Health Organization (2019) Global Tuberculosis Report 2019. World Health Organization, Geneva. https://www.who.int/tb/publications/global report/en/

[2] MacNeil, A., Glaziou, P., Sismanidis, C., Date, A., Maloney, S. and Floyd, K. (2020) Global Epidemiology of Tuberculosis and Progress toward Meeting Global Targets-Worldwide, 2018. Morbidity and Mortality Weekly Report, 69, 281-285. 
https://doi.org/10.15585/mmwr.mm6911a2

[3] World Health Organization (2020) Tuberculosis: Key Facts. World Health Organisation, Geneva. https://www.who.int/news-room/fact-sheets/detail/tuberculosis

[4] World Health Organization (2016) Global Tuberculosis Report. World Health Organisation, Geneva.

[5] Karumbi, J. and Garner, P. (2015) Directly Observed Therapy for Treating Tuberculosis. Cochrane Database of Systematic Reviews, 5, CD003343.

https://doi.org/10.1002/14651858.CD003343.pub4

[6] Asuquo, A.E., Pokam, T.B., Adindu, A., Ibeneme, E. and Obot, V. (2014) Health-Related Quality of Life (HRQOL) of Tuberculosis (TB) Patients in Akwa Ibom State, Nigeria. Journal of Tuberculosis Research, 2, 199-206.

https://doi.org/10.4236/jtr.2014.24024

[7] Bekang, F., Nsangou, T. and Ntone, R. (2016) Enquête cap sur la tuberculose. PNLT, 41.

[8] Kakhki, A.D. and Masjedi, M.R. (2015) Factors Associated with Health-Related Quality of Life in Tuberculosis Patients Referred to the National Research Institute of Tuberculosis and Lung Disease in Tehran. Tuberculosis and Respiratory Disease (Seoul), 78, 309-314. https://doi.org/10.4046/trd.2015.78.4.309

[9] McHorney, C.A., Ware Jr., J.E., Lu, J.F. and Sherbourne, C.D. (1994) The MOS 36-Item Short-Form Health Survey (SF-36): III. Tests of Data Quality, Scaling Assumptions, and Reliability across Diverse Patient Group. Medical Care, 32, 40-66. https://doi.org/10.1097/00005650-199401000-00004

[10] Ware, J.E., Kosinski, M., Dewey, J.E. and Gandek, B. (2001) How to Score and Interpret Single-Item Health Status Measures: A Manual For Users of the SF-8 Health Survey. Quality Metric Incorporated, Lincoln.

[11] Masumoto, S., Yamamoto, T., Ohkado, A., Yoshimatsu, S., Querri, A.G. and Kamiya, Y. (2014) Factors Associated with Health-Related Quality of Life among Pulmonary Tuberculosis Patients in Manila, the Philippines. Quality of Life Research, 23, 1523-1533. https://doi.org/10.1007/s11136-013-0571-x

[12] Marra, C.A., Marra, F., Cox, C.V., Palepu, A. and Fitzgerald, J.M. (2004) Factors Influencing Quality of Life in Patients with Active Tuberculosis. Health and Quality of Life Outcomes, 2, 58. https://doi.org/10.1186/1477-7525-2-58

[13] Wang, Y., Li, J. and Lu, F. (1998) Measuring and Assessing the Quality of Life of Patients with Pulmonary Tuberculosis. Chinese Journal of Tuberculosis and Respiratory Diseases, 21, 720-723. https://europepmc.org/article/med/11480072

[14] Louw, J., Peltzer, K., Naidoo, P., Matseke, G., Mchunu, G. and Tutshana, B. (2012) Quality of Life among Tuberculosis (TB), TB Retreatment and/or TB-HIV Co-Infected Primary Public Health Care Patients in Three Districts in South Africa. Health and Quality of Life Outcomes, 10, 77. https://doi.org/10.1186/1477-7525-10-77

[15] Chang, B., Wu, A.W., Hansel, N.N. and Diette, G.B. (2004) Quality of Life in Tuberculosis: A Review of the English Language Literature. Quality of Life Research, 13, 1633-1642. https://www.ncbi.nlm.nih.gov/pubmed/15651535 https://doi.org/10.1007/s11136-004-0374-1

[16] Kastien-Hilka, T., Rosenkranz, B., Sinanovic, E., Bennett, B. and Schwenkglenks, M. (2017) Health-Related Quality of Life in South African Patients with Pulmonary Tuberculosis. PLOS ONE, 12, e0174605. https://doi.org/10.1371/journal.pone.0174605

[17] Rajeswari, R., Muniyandi, M., Balasubramanian, R. and Narayanan, P.R. (2005) 
Perceptions of Tuberculosis Patients about Their Physical, Mental and Social Well-Being: A Field Report from South India. Social Science and Medicine, 60, 1845-1853. https://doi.org/10.1016/j.socscimed.2004.08.024

[18] Mamani, M., Majzoobi, M.M., Ghahfarokhi, S.M., Esna-Ashari, F. and Keramat, F. (2014) Assessment of Health-Related Quality of Life among Patients with Tuberculosis in Hamadan, Western Iran. Oman Medical Journal, 29, 102-105. https://doi.org/10.5001/omj.2014.25

[19] World Health Organization (2014) Basic Document. Geneva.

[20] Bauer, M.A., Leavens, K. and Schwartzman, A. (2013) Systematic Review and Meta-Analysis of the Impact of Tuberculosis on Health-Related Quality of Life. Quality of Life Research, 22, 2213-2235. https://doi.org/10.1007/s11136-012-0329-x

[21] Dhuria, M., Sharma, N. and Ingle, G.K. (2008) Impact of Tuberculosis on the Quality of Life. Indian Journal Community Medicine, 33, 58-59. https://doi.org/10.4103/0970-0218.39249

[22] Deribew, A., Tesfaye, M., Hailmichael, Y., Negussu, N., Daba, S., Wogi, A., Belachew, T., Apers, L. and Colebunders, R. (2009) Tuberculosis and HIV Co-Infection: Its Impact on Quality of Life. Health and Quality of Life Outcomes, 7, 105. https://doi.org/10.1186/1477-7525-7-105

[23] Freitas, I.M., Crispim, J.A., Pinto, I.C., Villa, T.C.S., Brunello, M.E.F., Pinto, P.F.P.S. and Arcêncio, R.A. (2012) Knowledge and Perception about Tuberculosis of Patients' Families under Directly Observed Treatment at a Health Service in Ribeirão Pretosp, Brazil. Florianópolis, 21, 642-649.

https://doi.org/10.1590/S0104-07072012000300020

http://www.scielo.br/pdf/tce/v21n3/en v21n3a20.pdf

[24] Raneswarj, R., Balaubramanian, R., Muniyandi, M., Geetharamani, S., Thresa, X. and Venkatesan, P. (1999) Socio-Economic Impact of Tuberculosis on Patients and Family in India. The International Journal of Tuberculosis and Lung Disease, 3, 869-877.

[25] Kahn, A., Walley, J., Newell, J. and Imdad, N. (2000) Tuberculosis in Pakistan: Socio-Cultural Constraints and Opportunities in Treatment. Social Science \& Medicine, 50, 247-254. https://doi.org/10.1016/S0277-9536(99)00279-8 